\title{
Bone metabolism and mineral density following renal transplantation
} György S Reusz, Attila J Szabó, Ferenc Péter, Éva Kenesei, Péter Sallay, Kay Latta,
Andras Szabó, Antal Szabó, Tivadar Tulassay
First Department of Paediatrics, Semmelweis University, Budapest, Hungary

G S Reusz

É Kenesei

P Sallay

A Szabó

A Szabó

Research Laboratory of the Hungarian

Academy of Sciences

A J Szabó

T Tulassay

Buda Children's Hospital, Budapest, Hungary

F Péter

Department of Paediatrics, Medical School, Hannover,

Germany

K Latta

Correspondence to: Dr G S Reusz, H-1083, Budapest, Bókay u. 53, Hungary

email: reusz@gyer1.sote.hu

Accepted 30 March 2000

\begin{abstract}
Aim-To study bone turnover following renal transplantation using a panel of biochemical markers and to correlate the results with both areal and volumetric bone mineral density (BMD).

Patients-A total of 31 patients aged 18.1 years were transplanted 5.4 years before this study. Control patients $(n=31)$ were age and gender matched.

Methods-In addition to measurement of biochemical markers, BMD was measured by single photon absorptiometry and peripheral quantitative computed tomography on the non-dominant radius. Results-Patients had reduced glomerular filtration rate, raised concentrations of serum phosphate, serum procollagene type I carboxy terminal propeptide, osteocalcin, and serum procollagene type I cross linked carboxy terminal telopeptide. The differences were still significant if only patients with normal intact parathyroid hormone were considered. BMD single photon absorptiometry $\mathrm{Z}$ score for age was significantly decreased. Following standardisation for height the differences were no longer present. With volumetric techniques patients had normal trabecular but decreased cortical and total BMD compared to age matched controls, but there was no difference from height matched controls.

Conclusion-Markers of bone turnover are increased following renal transplantation. However, the biochemical analysis did not allow conclusions to be drawn on the bone mineral content. BMD single photon absorptiometry $\mathrm{Z}$ score corrected for height and BMD measured by quantitative computed tomography compared to height matched controls were normal in paediatric renal transplantation patients. Height matched controls should be used in both areal and volumetric BMD measurements in states of growth failure. (Arch Dis Child 2000;83:146-151)
\end{abstract}

Keywords: bone mineral density; bone remodelling; bone turnover; renal transplantation

Diseases affecting the dynamics of metabolism of the growing skeleton may have long lasting sequelae in adulthood. There are conflicting data concerning bone mineral content in children and young adults following renal transplantation. Bone mineral content can be estimated by different methods of osteodensito- metry (single photon absorptiometry (SPA), dual energy $x$ ray absorptiometry (DEXA), and the newly introduced peripheral quantitative computed tomography (pQCT)). SPA and DEXA provide "linear" density data (expressed in $\mathrm{g} / \mathrm{cm}$ ), which can be transformed to "area" density by forming the ratio of the amount of mineral measured and the projected scanned area (expressed in $\mathrm{g} / \mathrm{cm}^{2}$ ). Based on the conventional linear and areal measurements it was generally accepted that severe osteopenia occurs following renal transplantation. ${ }^{12}$ The methods involving volumetric techniques ( $\mathrm{pQCT}$ ) indicate that mineral loss may be less significant and mineral content may even increase. ${ }^{3}$ However, bone densitometry does not give information about the underlying process of bone metabolism and the quality of bone formed. ${ }^{5}$

Bone metabolism is characterised by the opposite processes of formation and resorption resulting in continuous turnover. The equilibrium between formation and resorption will determine the gain, loss, or balance of total bone mass. ${ }^{67} \mathrm{~A}$ number of substances synthesised or released during bone remodelling are now available for clinical use. ${ }^{8}$ During remodelling the bone matrix becomes calcified. The currently used areal methods (SPA and DEXA) are fairly reliable and reproducible in adults. However, in children the influence of bone geometry on bone mineral density (BMD) measurements cannot be neglected. $^{4-11}$

Our aim in this cross sectional study was to assess and characterise the bone remodelling process by using a panel of markers of bone formation and resorption in children and young adults with functioning renal grafts for longer than one year. We assessed the relation of bone mineral density to bone markers using two different methods: SPA and pQCT.

\section{Patients and methods}

A total of 31 patients participated in the study. Table 1 shows relevant patient characteristics (and those of the control group).

Diagnoses in the renal transplantation group were: chronic pyelonephritis $(n=15)$ (combined with obstructive uropathy in 10 , and bilateral hypoplasia without obstruction in five), focal segmental glomerulosclerosis $(\mathrm{n}=4)$, tubulointerstitial nephritis $(\mathrm{n}=3)$, membranoproliferative glomerulonephritis $(n=3)$, nephronophtisis $(n=2)$, autosomal recessive polycystic kidney disease $(n=2)$, acute tubular necrosis $(n=1)$, and oligomeganephronia $(\mathrm{n}=1)$. 
Table 1 Clinical characteristics of the renal transplantation group and the controls

\begin{tabular}{|c|c|c|c|c|c|c|c|c|c|}
\hline & $n$ & Age (y) & Age range & $\begin{array}{l}\text { Tanner stage } \\
(1 / 2 / 3 / 4 / 5)\end{array}$ & $\begin{array}{l}\text { Height } \\
\text { (cm) }\end{array}$ & $\begin{array}{l}B M I \\
\left(\mathrm{~kg} / \mathrm{cm}^{2}\right)\end{array}$ & $\begin{array}{l}\text { Creatinine } \\
\text { ( } \mu \text { mol/l) }\end{array}$ & $\begin{array}{l}\text { Creatinine clearance } \\
\left(\mathrm{ml} / \mathrm{min} / 1.73 \mathrm{~m}^{2}\right)\end{array}$ & $\begin{array}{l}\text { Time since renal } \\
\text { transplantation }(y) \\
\text { (range) }\end{array}$ \\
\hline Renal transplantation & 31 & 18.1 & $8-26$ & $4 / 4 / 4 / 7 / 12$ & $148(5)$ & $20.2(0.9)$ & $142(11)$ & $65.9(3.9)$ & $5.4(1.6-11.2)$ \\
\hline Controls & 31 & 18.6 & $9-25$ & $2 / 2 / 3 / 7 / 17$ & $157(7)$ & $19.5(0.7)$ & $87(3)$ & $103.8(3.2)$ & - \\
\hline $\mathrm{p}$ value & - & NS & - & - & $\mathrm{p}=0.05$ & NS & $\mathrm{p}=0.001$ & $\mathrm{p}=0.001$ & - \\
\hline
\end{tabular}

Data are presented as mean (SEM).

BMI, body mass index.

Patients were on haemodialysis for a median of 2.5 years (range $0.5-5.5$ ) prior to transplantation. The mean age of the graft was 5.4 years at the time of the study (range 1.6-11.2). Basic immunosuppression consisted of combined cyclosporin A (mean dose of 3.6 (SEM 0.8) $\mathrm{mg} / \mathrm{kg} /$ day in two divided doses aiming at a trough level of $100-250 \mathrm{ng} / \mathrm{ml}$ ) and low dose (0.12 (0.2) $\mathrm{mg} / \mathrm{kg}$, once daily) methylprednisolone. Ten patients additionally received azathioprine (1.8 (0.4) mg/kg/day). All patients had stable renal function; no rejection had occurred for at least six months prior to this study.

Age and gender matched patients with no apparent nephrological disease undergoing minor surgical interventions (inguinal hernia, adenotomy, etc) were enrolled as controls.

Height was measured with a wall mounted stadiometer. Measurements were performed according to international guidelines ${ }^{12}$; reference data of normal children were obtained from the first Zurich Longitudinal Growth Study. ${ }^{13}$

Bone parameters measured were bone alkaline phosphatase (BAP), serum procollagene type I carboxy terminal propeptide (PICP), and osteocalcin (OC) for bone formation; and urinary pyridinoline and deoxypyridinoline crosslinks (PYD, DPD), and serum procollagene type I cross linked carboxy terminal telopeptide (ICTP) for bone reabsorption.

Blood samples were taken at the time of routine blood samples for these patients. The morning urine sample was used to assess urinary PYD and DPD concentration. Serum BAP and PICP was measured by enzyme linked immunosorbent assay (ELISA, Alkphase-B and Prolagen-C respectively, Metra Biosystems Inc., Mountain View, California). ICTP was measured by ELISA (CrossLaps, Osteometer Biotech Corp., Herlev, Denmark). Serum osteocalcin (bone Gla protein) was measured by ELISA (Osteocalcin, DAKO A/S Glostrup, Denmark).

Urinary PYD and DPD crosslinks were measured by HPLC (Crosslink HPLC, BioRad GmbH, München, Germany). The ratio of urinary PYD and DPD to urinary creatinine was used as measure of PYD and DPD excretion.

Intact (1-84) parathyroid hormone (iPTH) was determined by immunochemiluminometric two site assay (CIBA-CORNING, Fernwald, Germany). The reference range of the assay is $1-6 \mathrm{pmol} / 1 .^{14}$ All routine laboratory measurements (blood and urine calcium (Ca), inorganic phosphate $(\mathrm{P})$, and creatinine) were performed on a Technicon Autoanalyser.

Bone mineral density was assessed by both SPA and pQCT. SPA measurement was carried out using an osteodensitometer with radioiodine $\left.{ }^{125} \mathrm{I}\right)$ source (Gamma Works, Budapest, Hungary) at the distal third of the nondominant radius as described previously. ${ }^{14}$ The results were expressed as bone mineral content/ bone width ratio in $\mathrm{g} / \mathrm{cm}^{2}$ (bone mineral density, BMDspa). The coefficient of variation of the method was less than $4 \%$. BMDspa values of normal children $(n=251)$ determined previously were used as reference to calculate the standard deviation score (BMDspa $\mathrm{Z}$ score $).{ }^{15}{ }^{16}$ BMDspa $Z$ score was determined in two ways: firstly, BMDspa $Z$ score for age was calculated; then the values were corrected for height in order to eliminate bias caused by growth retardation. ${ }^{34917}$ PQCT was measured on the same arm as SPA by an XQCT-2000 device (Stratec Medizinische $\mathrm{GmbH}$, Pforzheim, Germany) at $4 \%$ of the forearm (ulnar) length proximal to the distal end of the radius. Total and spongiosa bone density at $45 \%$ core area of the bone was automatically analysed and reviewed for eventual erroneous area definition. The bone mineral density values for pQCT (BMDqct) were expressed in $\mathrm{mg} / \mathrm{cm}^{3}$. Coefficient of variation of the method was less than $2 \%$.

Patient data were compared to those of 31 age and gender matched controls. To assess the influence of short stature on the BMDqct values, the patient data were also compared to those of 31 height and gender matched controls. The controls for the pQCT data were taken from the database of the 251 children which also provided normal values for SPA measurements (Péter, unpublished data).

Table 2 Values of calcium, inorganic phosphate, iPTH, markers of bone remodelling, and bone mineral density of the renal transplantation patients and controls

\begin{tabular}{|c|c|c|c|c|c|c|c|c|c|}
\hline & $\mathrm{Ca}(\mathrm{mmol} / \mathrm{l})$ & $P(\mathrm{mmol} / \mathrm{l})$ & iPTH (pmol/l) & $B A P(U / l)$ & $P I C P(\mu g / l)$ & $O C(\mu g / l)$ & $\begin{array}{l}\text { PYD } \\
(\text { (umol/mmol) }\end{array}$ & $\begin{array}{l}\text { DPD } \\
(\mu \mathrm{mol} / \mathrm{mmol})\end{array}$ & $\operatorname{ICTP}(\mathrm{pmol} / \mathrm{l})$ \\
\hline Renal transplantation & $2.43(0.05)$ & $1.66(0.1)$ & $12.9(4.4)$ & $65(11)$ & $335(55)$ & $26.9(2.9)$ & $221(37)$ & $44.3(6.6)$ & $11.6(1.4)$ \\
\hline Controls & $2.42(0.03)$ & $1.49(0.04)$ & $2.0(0.2)$ & $48(8)$ & $190(24)$ & $18.0(3.4)$ & $232(49)$ & $34.7(6.3)$ & $6.3(0.7)$ \\
\hline $\mathrm{p}$ value & NS & $\mathrm{p}=0.01$ & $\mathrm{p}=0.03$ & NS & $\mathrm{p}=0.02$ & $\mathrm{p}=0.03$ & NS & NS & $\mathrm{p}=0.01$ \\
\hline
\end{tabular}

Data are presented as mean (SEM).

$\mathrm{Ca}$, total serum calcium; $\mathrm{P}$, inorganic phosphate; iPTH, intact parathyroid hormone; BAP, bone alkaline phosphatase; OC, osteocalcin; PICP, serum procollagene type I carboxy terminal propeptide; ICTP, procollagene type I cross linked carboxy terminal telopeptide (ICTP); PYD, urinary pyridinoline; DPD, urinary deoxypyridinoline crosslinks. 
Table 3 Correlations ( $r$ values) between height, weight, inorganic phosphate, and bone parameters in the renal transplantation group

\begin{tabular}{llllllllll}
\hline & Age & Height & $P$ & BAP & PICP & OC & PYD & DPD & ICTP \\
\hline Age & 1 & 0.74 & $-0.45^{\star}$ & $-0.59^{\star \star}$ & $-0.63^{\star \star}$ & $-0.61^{\star \star}$ & $-0.81^{\star \star}$ & $-0.78^{\star \star}$ & $-0.46^{\star}$ \\
Height & & 1 & $-0.53^{\star}$ & $-0.58^{\star \star}$ & $-0.55^{\star}$ & $-0.62^{\star \star}$ & $-0.75^{\star \star}$ & $-0.66^{\star \star}$ & -0.059 \\
\hline
\end{tabular}

${ }^{\star} \mathrm{p}<0.05 ;{ }^{\star \star} \mathrm{p}<0.01$.

$\mathrm{P}$, inorganic phosphate; $\mathrm{BAP}$, bone alkaline phosphatase; OC, osteocalcin; PICP, serum procollagene type I carboxy terminal propeptide; ICTP, procollagene type I cross linked carboxy terminal telopeptide (ICTP); PYD, urinary pyridinoline; DPD, urinary deoxypyridinoline crosslinks.

Table 4 Correlations ( $r$ values) between the bone parameters

\begin{tabular}{lllllll}
\hline & BAP & PICP & OC & PYD & DPD & ICTP \\
\hline BAP & 1 & $0.58^{\star}$ & $0.83^{\star \star}$ & $0.63^{\star \star}$ & $0.54^{\star}$ & $0.78^{\star \star}$ \\
PICP & & 1 & $0.65^{\star}$ & $0.80^{\star \star}$ & $0.79^{\star \star}$ & 0.42 \\
OC & & & 1 & $0.80^{\star \star}$ & $0.71^{\star}$ & $0.56^{\star}$ \\
PYD & & & & 1 & $0.96^{\star \star}$ & 0.46 \\
DPD & & & & & 1 & 0.36 \\
ICTP & & & & & & 1 \\
\hline
\end{tabular}

${ }^{\star} \mathrm{p}<0.05 ;{ }^{\star \star} \mathrm{p}<0.01$

BAP, bone alkaline phosphatase; OC, osteocalcin; PICP, serum procollagene type I carboxy terminal propeptide; ICTP, procollagene type I cross linked carboxy terminal telopeptide (ICTP); PYD, urinary pyridinoline; DPD, urinary deoxypyridinoline crosslinks.

Statistical analysis was performed by the Mann-Whitney U test and linear regression analysis using the Statistica 5.1 (StatSoft) software package.

The study was performed according to the Declaration of Helsinki and was approved by the local ethical committee.

\section{Results}

As presented in table 1, patients and controls were matched for age. The renal transplantation patients were significantly shorter than the matched controls. Renal transplantation patients had significantly higher creatinine and correspondingly lower creatinine clearance values than the controls. Table 2 shows the values of serum $\mathrm{Ca}, \mathrm{P}, \mathrm{iPTH}$, and markers of bone remodelling.

Serum Ca was normal. Serum P, iPTH, PICP, OC, and ICTP were significantly increased. The rise of BAP and DPD did not reach significance. If only those patients ( $\mathrm{n}=21)$ with normal iPTH $(<6.0 \mathrm{pmol} / \mathrm{l})$ were considered, serum creatinine was still greater than normal $(130 \mu \mathrm{mol} / 1, \mathrm{p}<0.001)$. Further, PICP, OC, and ICTP concentrations were still significantly greater than those of the controls (366 (69) $\mu \mathrm{g} / 1, \quad \mathrm{p}<0.02 ; 24.7$ (2.1) $\mu \mathrm{g} / \mathrm{l}$, $\mathrm{p}<0.05 ; 10.3(1.2) \mathrm{pmol} / \mathrm{l}, \mathrm{p}<0.01$, respectively).

If patients with accelerated pubertal growth (Tanner score 2, 3, and 4) were excluded, the remaining patients with Tanner stages 1 and 5
( $\mathrm{n}=20)$ still had significantly higher PICP, OC, and ICTP values than the non-pubertal controls (310 (52) $\mu \mathrm{g} / 1, \mathrm{p}<0.03 ; 19.1$ (2.9) $\mu \mathrm{g} / 1, \quad \mathrm{p}<0.05 ; 7.4$ (1.1) $\mathrm{pmol} / \mathrm{l}, \quad \mathrm{p}<0.05$, respectively).

In both controls and renal transplantation patients, serum $\mathrm{P}$ and the bone markers were closely and negatively correlated with age and height (table 3). Close and significant positive correlation was observed between almost all the bone markers measured (table 4)

There was no correlation between iPTH and age or the anthropometric parameters (height, weight, BMI). In renal transplantation there was a negative correlation between creatinine clearance and the time since transplantation $(r=-0.61 ; \mathrm{p}<0.001)$. iPTH was closely and positively related to serum creatinine and negatively to creatinine clearance $(r=0.49$ and -0.49 respectively; $\mathrm{p}<0.01)$ and negatively correlated to serum calcium $(r=-0.48$; $\mathrm{p}<0.01)$. However, there was no correlation between iPTH and any of the bone markers measured.

The BMDspa and the age matched BMDspa $\mathrm{Z}$ data for renal transplantation patients were significantly below those of the controls. Following correction of BMDspa $\mathrm{Z}$ for height, the differences were no longer present (table 5).

Total and cortical BMDqct was significantly lower in the renal transplantation group compared to the age matched controls. However (as shown in table 1) transplanted patients had a significant height deficit. Thus, compared to height matched controls the differences were no longer present.

The absolute value of BMDspa of the distal radius correlated to age $(r=0.53, \mathrm{p}<0.01)$ and height $(r=0.46, \mathrm{p}<0.02)$. BMDqct of the cortical bone correlated positively with age $(r=0.39, \mathrm{p}<0.05)$, and height $(r=0.38$; $\mathrm{p}<0.05)$. No correlation could be found between trabecular density and age. There was a positive correlation between the absolute value of BMDspa and the cortical volumetric BMDqct $(r=0.39, \mathrm{p}<0.04)$ in renal trans-

Table 5 Values of bone mineral density measured by SPA and $p Q C T$ in transplanted patients and controls

\begin{tabular}{|c|c|c|c|c|c|c|c|c|c|c|c|}
\hline & \multicolumn{3}{|l|}{$S P A$} & \multicolumn{8}{|l|}{$p Q C T$} \\
\hline & \multirow{2}{*}{$\begin{array}{l}\text { BMDspa } \\
\left(\mathrm{g} / \mathrm{cm}^{2}\right)\end{array}$} & \multirow{2}{*}{$\begin{array}{l}\text { BMDspa } Z \\
\text { for age }\end{array}$} & \multirow{2}{*}{$\begin{array}{l}\text { BMDspa } Z \text { for } \\
\text { height }\end{array}$} & \multicolumn{3}{|c|}{ Controlled for age $\left(\mathrm{mg} / \mathrm{cm}^{3}\right)$} & \multicolumn{5}{|c|}{ Controlled for height $\left(\mathrm{mg} / \mathrm{cm}^{3}\right)$} \\
\hline & & & & Total & Trab & Cort & Height & Age & Total & Trab & Cort \\
\hline $\begin{array}{l}\text { Renal } \\
\quad \text { transplantation }\end{array}$ & $\begin{array}{l}0.589 \\
(0.09)\end{array}$ & $\begin{array}{l}-2.237 \\
(0.049)\end{array}$ & $\begin{array}{l}-0.2772 \\
(0.1014)\end{array}$ & $298(12)$ & $199(20)$ & $367(15)$ & $148(5)$ & $18.1(2.9)$ & $298(12)$ & $199(20)$ & $367(15)$ \\
\hline Controls & $\begin{array}{l}0.7910 \\
(0.07)\end{array}$ & $\begin{array}{l}-0.1462 \\
(0.097)\end{array}$ & $-0.1174(0.761)$ & $324(11)$ & $202(6)$ & $423(20)$ & $149(8)$ & $12.3(2.5)$ & $296(5)$ & $190(5)$ & $384(6)$ \\
\hline $\mathrm{p}$ value & $\mathrm{p}=0.001$ & $\mathrm{p}=0.01$ & NS & $\mathrm{p}=0.05$ & NS & $\mathrm{p}=0.02$ & NS & $\mathrm{p}=0.001$ & NS & NS & NS \\
\hline
\end{tabular}

Data are presented as mean (SEM).

SPA, single photon absorptiometry; pQCT, peripheral quantitative computed tomography; BMDspa, bone mineral density measured by SPA; BMDspa Z, bone mineral density standard deviation $(Z)$ score; Total, total bone mineral density; Trab, trabecular bone mineral density; Cort, cortical bone mineral density. 
plantation. The change of BMDspa with age was much higher than that of cortical BMD. Uncorrected BMDspa was 25\%; BMDqct was $9 \%$ lower than the corresponding control.

No further correlation could be found between any of the SPA and pQCT data. There was no correlation between areal or volumetric BMD (BMDspa and BMDqct) values and iPTH or creatinine clearance. There was no correlation between any of the measured bone markers and any of the BMD values measured or calculated.

\section{Discussion}

This study used a panel of bone markers and two different methods of bone densitometry in order to characterise bone metabolism and mineralisation in transplanted children and young adults.

Until recently it was generally accepted that most patients who had received renal transplantation in childhood had moderate to severe osteopenia, even many years after renal transplantation. ${ }^{12}$ However, most of those studies using linear or areal BMD measurements did not take into account the problems of interpretation caused by growth dependent changes. Accordingly, recent data indicate that following a significant decrease in BMD during the first year after transplantation, BMD increases significantly; weight/height related or volumetric $\mathrm{BMD}$ is not subsequently reduced. ${ }^{349}$ On the other hand, combined densitometric and histological studies of bone structure following transplantation suggested that normal BMD values do not exclude the presence of pathological bone structure, including high turnover, adynamic, and/or mixed bone disease. Bone remodelling following transplantation could be influenced by the actual renal function, parathyroid hyperplasia, the effects of immunosuppressive drugs, and the stage of puberty. ${ }^{35911}$

Although histological analysis of the iliac bone biopsy is the gold standard for assessing bone structure and remodelling, ${ }^{511}{ }^{18}$ the procedure is not routinely performed and considerable efforts have been undertaken to develop reliable non-invasive methods to characterise bone turnover. A number of non-invasive markers representative of different steps of bone metabolism are now available for clinical use. ${ }^{8}$ Using a broad panel of those markers we have found both an increased activity of bone formation and resorption. Formation was measured by BAP and OC as indicators of osteoblast activity and PICP which results from the extracellular cleavage of a molecule of type I procollagene at the moment of its incorporation into the bone matrix. ${ }^{19} \mathrm{OC}$ and PICP were significantly raised. Interestingly the elevation of BAP - regarded as one of the most sensitive markers of formation - did not reach the level of significance.

Quantitative analysis of PYD and DPD in urine as well as ICTP in serum containing cross linking molecules has been shown to serve as an excellent marker of bone resorption rate in a great number of diseases influencing bone. ${ }^{820}$ We found a non-significant rise in
DPD ( $p<0.08)$. ICTP was significantly increased. Although ICTP tends to accumulate in serum with the decline in renal function, there was no correlation between ICTP and ECC in our renal transplantation patients, suggesting that the accumulation occurs by creatinine clearance values higher than observed in this study. ${ }^{21}$

As expected, all markers studied presented an age dependent course. To get round this problem, patient data were compared to age and gender matched controls. Even with this approach, there was some difference in pubertal maturation between the patient and control group. However, if only patients with stages 1 and 5 were considered the differences still persisted.

We considered the possible role of actual renal function: patients participating in this cross sectional study were in "steady state", thus had stable, but somewhat decreased creatinine clearance, no recent episode of rejection, and standard immunosuppressive treatment. The impairment of creatinine clearance was correlated with time since transplantation, in accordance with data indicating a slow and gradual loss of graft function due mainly to non-immunological reasons. ${ }^{22} \mathrm{Hy}-$ perphosphataemia accompanied by the increased iPTH measured could well be one reason for high bone turnover. ${ }^{83}$ However, there was no correlation between iPTH and any of the bone markers, or BMD data measured or calculated. Further, PCIP, OC, and ICTP were still significantly increased if only patients having $\mathrm{PTH}$ in the normal range were included. Thus iPTH alone could not account for the increased turnover.

Cyclosporin has been reported to increase both bone formation and resorption in the rat and to reduce cancellous bone volume. ${ }^{1124}$ Similar changes have been described in bone biopsy specimens of adult and paediatric renal transplant recipients. ${ }^{511} 25$ All patients received cyclosporin plus methylprednisolone, ten patients having additionally azathioprine as basic immunosuppression. Thus cyclosporin is likely to account for higher rates of bone formation in patients with normal serum iPTH concentrations, as prednisolone and other corticosteroids are usually associated with decreases rather than increases in bone formation and turnover. ${ }^{26}$ Chronic steroid treatment even in higher doses, did not cause substantial changes in the level of bone markers in an unpublished study in patients with steroid treated nephrotic syndrome (Reusz, unpublished data). Thus, it is unlikely that the low dose treatment of the renal transplantation group would contribute to the biochemical changes observed. The role of other immunosuppressive agents as potential modifiers of bone resorption has not been completely evaluated; however, azathioprine does not seem to affect bone mineral density by itself during long term immunosuppressive treatment for reasons other than renal transplantation. ${ }^{1127}$

The lack of correlation between markers of bone turnover and the BMD data may seem disappointing. However, in a recent study even 
a detailed histological evaluation failed to show correlation between the histopathological subtypes of bone pathology and BMD, showing the complexity of the problem. ${ }^{5}$ The markers of bone formation and degradation were all closely interrelated. This only signifies that formation and degradation are coupled processes. In future studies, comparison of the rise of the curves representing the single correlations measured in different patient groups and controls could theoretically provide data on the shift towards formation or resorption in the groups studied.

Interpretation of the $\mathrm{BMD}$ values in renal transplantation needs caution. Because areal BMD data are influenced by bone geometry dependent of patient height, ${ }^{810}$ the significantly lower BMDspa and BMDspa $\mathrm{Z}$ for age found should not necessarily be interpreted as defective mineralisation. Renal transplantation patients were significantly shorter than age matched controls, a difference that could be attributed to the growth failure caused by uraemia and chronic steroid treatment. Correspondingly, if corrected for height the BMD Z values were almost normal, indicating that most of the "mineral deficit" could be attributed to the reduced age related geometry of the bones rather than to a really lower mineral content. These data measured on a structurally different bone than the studies quoted (radius versus lumbar spine ${ }^{4}{ }^{9}$ ) indicate that the need for height correction is a more general rule valid for different types of bones.

Using the volumetric method of pQCT, renal transplantation patients had a significant deficit of total and cortical, but not of trabecular mineral content, compared to age matched controls. However, the difference virtually disappeared when patients were compared to height matched controls. The normal trabecular mineral density measured by pQCT is compatible with the findings of Feber $e t$ al and Klaus et al who measured (the mainly trabecular) density of the lumbar spine and corrected it for vertebral volume or height respectively. ${ }^{4} 9$ The deficit in the total and cortical BMD of renal transplantation measured by pQCT could be attributed to the fact that the age matched control group had an $8.6 \%$ higher total and a $9.2 \%$ higher cortical bone density than the six years younger height matched control group. Although volumetric BMD is much less dependent on age than linear or areal methods, it reflects however the small gain in mineral content observed also by measuring the percentage of bone ash of the different bones of the skeleton. ${ }^{10}{ }^{28}$ This may be due in part to the age dependent development of the musculoskeletal unit described recently showing a close relation between muscular strength and BMD of the underlying bones. ${ }^{10} 2930$ This could also explain the correlation between BMDspa and cortical BMDqct. However, because BMDspa is also influenced by height (and thus bone geometry), changes of BMDspa with age were much more expressed than that of cortical BMDqct.
We conclude that measurements of parameters of bone formation and degradation are indicative of an increased bone turnover in renal transplantation patients. However, analysis of the single parameters did not allow conclusions on bone mineral content. The "snapshot" given by the bone markers does not necessarily reflect the long lasting process of bone mineral deposition reflected by the mineral content measured.

BMD Z score corrected for height and volumetric BMD measured by pQCT compared to height matched controls are normal in paediatric patients studied 5.4 (range 1.6-11.2) years after renal transplantation. Height matched controls should be used in both areal and volumetric BMD measurements in all states of growth deficit.

Parts of the study were supported by the FKFP (1136/97), OTKA (T-22829, T-23850, T-31986, T-31950, F29782) OMFB (TéT D-5/1998), and ETT (511/96, 80-4/98) grants.

1 Chesney RW, Rose PG, Mazess RB. Persistence of diminished bone mineral content following renal transplandiminished bone mineral content following renal

2 Boot AM, Nauta J, Hokken-Koelaga ACS, et al. Renal ransplantation and osteoporosis. Arch Dis Child 1995;72: $502-6$.

3 Schönau E, Wentzlik U, Michalk D, Scheidhauer K. Is there an increase of bone density in children? Lancet 1993;342: $689-90$

4 Klaus G, Paschen C, Wüster C, et al. Weight/height related bone mineral density is not reduced after renal transplantation. Pediatr Nephrol 1998;12:343-34.

5 Cueto-Manzano AM, Konel Sh, Hutchinson AJ, et al. Bone loss in long-term renal transplantation: histopathology and densitometry analysis. Kidney Int 1999;55:2021-9.

6 Termine J. Bone matrix proteins and the mineralization process. In: Favus M, ed. Primer on the metabolic bone process. In: Favus $M$, ed. Primer on the metabolic bone
diseases and disorders of mineral metabolism. New York: Raven Press, 1993:21-5.

7 Baron R. Anatomy and ultrastructure of bone. In: Favus M, ed. Primer on the metabolic bone diseases and disorders of min ral metabolism. New York: Raven Press, 1993:3-9.

8 Urena P, Vernejoul MC. Circulating biochemical markers of bone remodeling in uremic patients. Kidney Int 1999;55: 2141-56.

9 Feber J, Cochat P, Braillon P, et al. Bone mineral density after renal transplantation in children. F Pediatr 1994;125: $870-5$.

10 Schönau E. Problems of bone analysis in childhood and adolescence. Pediatr Nephrol 1998;12:420-9.

11 Sanchez CP, Salusky I, Kuizon BD, et al. Bone disease in children and adolescents undergoing successful renal transplantation. Kidney Int 1998;53:1358-64.

12 Cameron N. The measurement of human growth. London: Croom Helm, 1984

13 Prader A, Largo RH, Molinari L, Issler C. Physical growth of Swiss children from birth to 20 years of age. Helv Paediatr Acta 1989;43(suppl 52):1-125.

14 Reusz GS, Dobos M, Vásárhelyi B, et al. Sodium transport and bone mineral density in hypercalciuria with thiazide treatment. Pediatr Nephrol 1998;12:30-4

15 Péter F, Kántor I, Blatniczky L, et al. Radial bone mineral density (BMD) in Hungarian children and adolescents. In: Schõnau E, ed. New trends and diagnostic possibilities in paediatric osteology. Amsterdam: Elsevier, 1995:83-91.

16 Péter F, Muzsnai Á, Kántor I. Thyroid hormones and bone mineralization in children and adolescents. In: Schönau E, Matkovic V, eds. Paediatric osteology. Prevention of osteoporosis - a paediatric task? Amsterdam: Elsevier, 1998: $183-9$.

17 Reusz GS, Stubnya G, Horváth CS, et al. X-linked hypophosphatemia: effects of treatment with recombinant
human growth hormone. Pediatr Nephrol 1997;11:573-7.

18 Parfitt AM, Drezner MX, Glorieux FH, et al. Bone histomorphometry: standardization of nomenclature, symbols and units. F Bone Miner Res 1987;2:595-610

19 Hamdy NAT, Risteli J, Risteli S, et al. Serum type I procollagene peptide: a non-invasive index of bone formation in patients on hemodialysis? Nephrol Dial Transplant 1994;9: 511-16.

20 Branca F, Robins SP, Ferro-Luzzi A, Golden MHN. Bone turnover in malnourished children. Lancet 1992;340:14936.

21 Mazzafero S, Pasquali M, Ballanti P, et al. Diagnostic value of serum peptides of collagen synthesis and degradation in dialysis renal osteodystrophy. Nephrol Dial Transplant 1995; 10:52-8.

22 Szabo A, Heemann U. Ischemia/reperfusion and chronic allograft rejection. Transplant Proc 1998;30:4281-4. 
23 Salusky IB, Ramirez JA, Oppenheim WL, et al. Biochemical markers of renal osteodystrophy in pediatric patients ;5:253-8.

24 Del Pozo E, Lippuner K, Ruch W, et al. Different effects of cyclosporin-A on bone remodeling in young and adult rats. Dial Transplant Nephrol 1995;16:271S-5S

25 Bourbigot B, Moal MC, Cledes J. Bone histology in renal transplant patients receiving cyclosporin. Lancet 1999;1 1048-9.

26 Lukert BP, Raisz LG. Glucocorticoid-induced osteoporosis: pathogenesis and management. Ann Intern Med 1990;112: 352-64.
27 Floren $\mathrm{CH}$, Ahren B, Bengtsson M, et al. Bone mineral density in patients with Crohn's disease during long-term 28 Trotter M, Hixon BB. Sequential changes in weight density, and percentage ash weight of human skeletons from early fetal period through old age. Anat Rec 1973;179:1-18.

29 Spindler A, Paz S, Berman A, et al. Muscular strength and bone mineral density in hemodialysis patients. Nephrol Dial Transplant 1997;12:128-32.

30 Schönau E. The development of the skeletal system in children and the influence of muscular strength. Horm Res $1997 ; 49: 27-31$.

\section{Sneha-India and the International Council for Research into the Fetal Origins of Adult Disease}

\section{First World Congress: Fetal Origins of Adult Disease}

Sponsored by the British Medical fournal

2-4 February 2001, Mumbai, India

Poor fetal growth is associated with an increased risk of adult cardiovascular disease and diabetes, which has led to the hypothesis that these disorders originate through undernutrition in utero. Evidence also links fetal growth with later osteoporosis, neurological and psychiatric disease, hormone related cancers and atopy. This conference will bring together, for the first time, clinicians, epidemiologists, and basic scientists working in this field.

Topics include:

Cardiovascular disease

Diabetes

Cancer

Osteoporosis

Asthma

Ageing

Mental Health

Maternal nutrition

Control of fetal growth

Hormonal programming

Immune function

Strategies for preventing disease

Plenary speakers include: Claude Lenfant (USA), Nick Hales (UK), Christopher Martyne (UK), Chittaranjan Yajnik (India), Michael Meaney (Canada), Jeffrey Robinson (Australia), Jane Harding (New Zealand), Kent Thornburg (USA), John Challis (Canada), Alan Jackson (UK), Keith Godfrey (UK), Patrick Bateson (UK), Peter Gluckman (New Zealand).

Scientific committee chairman: David Barker, Southampton, UK

Organising committee chairman: Anand Pandit, Pune, India

Further details from: Ms Alifiya S Motiwala (tel: +91 22651 6439/645 6763; fax: +91 22651 6438; email:mrcssc@vsnl.com).

Alternatively, fill in the "Yes, I am interested" reply slip on our website: www.sneha-india.org 